\title{
LV-OP-2-2
}

\section{Development and validation of an individualized prediction calculator of postoperative mortality within 6 months after surgical resection for hepatocellular carcinoma: An international multicenter study}

\author{
Lei LIANG ${ }^{1}$, Bing QUAN² , Yong-Kang DIAO', Chao LI ${ }^{3}$, Ming-Da WANG ${ }^{3}$, Wan Yee LAU ${ }^{4}$, Cheng-Wu ZHANG ${ }^{1}$, \\ Timothy M. PAWLIK ${ }^{5}$, Dong-Sheng HUANG ${ }^{1}$, Feng SHEN ${ }^{3}$, Tian YANG ${ }^{* 3}$ \\ 'Department of Hepatobiliary, Pancreatic and Minimal Invasive Surgery, Zhejiang Provincial People's Hospital, \\ People's Hospital of Hangzhou Medical College, Hangzhou, China \\ ${ }^{2}$ Department of Clinical Medicine, Second Military Medical University (Navy Medical University), Shanghai, China \\ ${ }^{3}$ Department of Hepatobiliary Surgery, Eastern Hepatobiliary Surgery Hospital, \\ Second Military Medical University (Navy Medical University), Shanghai, China \\ ${ }^{4}$ Faculty of Medicine, The Chinese University of Hong Kong, Hong Kong, China \\ ${ }^{5}$ Department of Surgery, Ohio State University, Wexner Medical Center, Columbus, OH, USA
}

Introduction: Evidence-based decision-making is critical to optimize the benefits and mitigate futility associated with surgery for patients with malignancies. Untreated hepatocellular carcinoma (HCC) has a median survival of only 6 months. The objective was to develop and validate an individualized patient-specific tool to predict preoperatively the benefit of surgery to provide a survival benefit of at least 6 months following resection.

Methods: Using an international multicenter database, patients who underwent curative-intent liver resection for HCC from 2008 to 2017 were identified. Using random assignment, two-thirds of patients were assigned to a training cohort with the remaining onethird assigned to the validation cohort. Independent predictors of postoperative death within 6 months after surgery for HCC were identified and used to construct a nomogram model with a corresponding online calculator. The predictive accuracy of the calculator was assessed using C-index and calibration curves.

Results: Independent factors associated with death within 6 months of surgery included age, Child-Pugh grading, portal hypertension, alpha-fetoprotein level, tumor rupture, tumor size, tumor number and gross vascular invasion. A nomogram that incorporated these factors demonstrated excellent calibration and good performance in both the training and validation cohorts (C-indexes: 0.802 and 0.798 ). The nomogram also performed better than four other commonly-used HCC staging systems (C-indexes: 0.800 vs. 0.542-0.748).

Conclusions: An easy-to-use online prediction calculator was able to identify patients at highest risk of death within 6 months of surgery for HCC. The proposed online calculator may help guide surgical decision-making to avoid futile surgery for patients with HCC. 